\title{
Three-dimensional crack growth modelling of a Ni-based superalloy at elevated temperature and sustained loading
}

Erik Storgärds, Kjell Simonsson and Sören Sjöström

\author{
Linköping University Post Print
}

\section{Tweet}

N.B.: When citing this work, cite the original article.

Original Publication:

Erik Storgärds, Kjell Simonsson and Sören Sjöström, Three-dimensional crack growth modelling of a Ni-based superalloy at elevated temperature and sustained loading, 2016, Theoretical and applied fracture mechanics (Print).

http://dx.doi.org/10.1016/j.tafmec.2015.11.008

Copyright: Elsevier

http://www.elsevier.com/

Postprint available at: Linköping University Electronic Press

http://urn.kb.se/resolve?urn=urn:nbn:se:liu:diva-121004

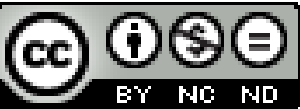




\title{
Three-dimensional crack growth modelling of a Ni-based superalloy at elevated temperature and sustained loading
}

\author{
Erik Storgärds $^{\mathrm{a}, *}$, Kjell Simonsson ${ }^{\mathrm{a}}$, Sören Sjöström ${ }^{\mathrm{a}}$ \\ ${ }^{a}$ Division of Solid Mechanics, Department of Management and Engineering, Linköping University, SE-58183 \\ Linköping, Sweden
}

\begin{abstract}
High temperature materials subjected to elevated temperature have been shown to be sensitive to dwell times, giving an increased crack growth rate. The interaction between these dwell times and rapid cyclic loads have been shown to constitute a complex problem. Many models have been developed for 1D conditions, but the application to general 3D conditions has seldom been seen, although this is the most common case in most structures. In this paper a model for taking care of the interaction between these load modes in general 3D crack growth has been developed. The model uses 1D results for extension to general 3D, thus providing for local crack front evolution with a minimum of numerical simulations. Consequently, a history dependent crack growth law in 3D is given in this paper, which is capable of tracking the damage from the sustained load, and the interaction with rapid cyclic loading. The model has been implemented for usage with finite element calculations and several different tests are simulated and compared with experimental results for the nickel based superalloy Inconel 718 at $550^{\circ} \mathrm{C}$. The simulation results show crack shapes in agreement with experimental fracture surfaces and time to failure.

Keywords: Sustained load, Crack growth modelling, Crack tunnelling, Ni-based superalloy, High temperature
\end{abstract}

\footnotetext{
${ }^{*}$ Corresponding author. Tel: $+46(0) 13282475$

Email address: erik. storgards@liu.se (Erik Storgärds)
} 


\section{Introduction}

Life prediction in gas turbine applications presents a challenging task as high temperatures in combination with severe thermo-mechanical loads push the materials to their degradation limit. Load cycles that a gas turbine is exposed to typically include a start-up phase with long and strong thermal transients, followed by a block of constant power at which the turbine may be subjected to various cyclic loads, e.g. caused by turbulence or fluctuations on the power grid. After this, a shutdown phase is usually initiated with slow ramp-down to idle condition. The interaction between rapid cyclic and sustained load, either at constant power or during long start-up and shut-down phases, can exert a major impact on crack growth rates, and thus on the life of critical components.

In order to handle load cycles of the type discussed above, a number of different models have been developed, which are either linear superposition models of the two load modes, see e.g. [1, 2], or models with interactive behaviour, cf. [3]. Independently of the choice of model the underlying foundation is, in the majority of cases, based on physical observations from crack paths. Cracks exposed to sustained load have been seen to fracture in an intergranular mode rather than the common transgranular cracking, see e.g. [4-6]. The different observations have developed into two damage mechanism theories of how the grain boundaries are affected. These are, stress accelerated grain boundary oxidation (SAGBO) and dynamic embrittlement (DE), see e.g. [7, 8]. In tests in vacuum the effect of sustained load damage was not observed, see e.g. [8], by which it may be concluded that grain boundary damage is in need of some kind of embrittling element e.g. oxygen to function.

Sustained load damage has also been paid more quantitative attention to, e.g. $[9,10]$ where Inconel 718 corner cracks were investigated. The findings on these fracture surfaces show another important behaviour which is also seen for cyclic loads, namely crack tunnelling (more growth in the depth than on the surface). It is, as concluded by numerous authors for the case of cyclic load, closely related to the crack constraint over the front, see e.g. [11] and for some more recent findings, see e.g. [12-14], where it is shown how plane strain and plane stress conditions will have a pronounced effect 
on the crack shape. Crack tunnelling with cyclic loads may therefore be related to the amount of crack closure present, as higher closure values will be associated with more pronounced plane stress conditions and thus give a lower propagation rate $[15,16]$. Tunnelling related to sustained load damage on the other hand, cannot for obvious reasons be related to crack closure but must still be dependent on constraint level, cf. $[9,17]$.

Many authors have developed models to describe the tunnelling, e.g. in [18] where the SIF value was scaled by a factor depending on the load ratio, in [19] where the constant $\mathrm{C}$ in Paris law is reduced over the crack front, and in [20] where a minimisation of errors of the growth rates are used to produce the correct crack shape. Studies of the actual constraint along the crack front have also been carried out by numerous authors. These simulations are usually based on dissolving the stress at the crack tip along the crack front to, e.g., determine the triaxial stress values along the front, see e.g. [21] or to simulate 3D crack closure, see e.g. [22]. Examples such as these show the difficulty of describing a correct constraint relation; the latter alternative ([21, 22]) suffering from heavy mesh dependence when trying to dissolve the behaviour at the crack tip, but providing for more freedom in crack shape, loads etc.; the former alternative ([18-20]) being restricted to specified analytical cases, but on the other hand providing for rapid prediction results. Finding a method between these two should therefore be of high interest, especially for use in an industrial context.

Exploring the possibilities to describe the correct crack shape evolution during sustained load with interaction of cyclic load, will be of great interest. In this paper a modelling approach for describing crack shape evolution under these load modes is developed for 3D crack growth applications, and is validated for in-plane loaded surface crack test specimens of the Ni-based superalloy Inconel 718 at $550{ }^{\circ} \mathrm{C}$. The model developed takes its form from the damage evolution (embrittling of grain boundaries) for sustained load and interaction with cyclic load. By using the already developed damaged zone model $[3,23]$ for $1 \mathrm{D}$, and extending it further into full 3D, an efficient load history dependence can be reached. Detailed numerical simulations have been carried out for both cyclic loads and sustained loads, as well as for mixed cyclic and dwell time loading. 


\section{Nomenclature}

$\begin{array}{llll}A & \text { fitting parameter } & \text { SAGBO } & \text { stress accelerated grain boundary oxidation } \\ B & \text { fitting parameter } & \text { SIF } & \text { stress intensity factor } \\ C & \text { power law constant } & a & \text { crack length } \\ D & \text { damaged zone length } & \dot{m} & \text { damage mechanism based growth rate } \\ \text { DCPD } & \text { direct current potential drop } & n & \text { power law constant } \\ \text { DE } & \text { dynamic embrittlement } & \alpha & \text { constraint parameter } \\ \text { E } & \text { fitting parameter } & \Delta K & \text { stress intensity factor range } \\ \text { EDM } & \text { electro discharge machining } & \sigma & \text { stress } \\ K & \text { stress intensity factor } & R & \text { load ratio } \\ \text { S } & \text { scale function } & & \\ \text { Subscripts } & & & \\ \text { c } & & & \\ \text { t } & \text { cyclic value } & & \end{array}$

\section{Experiments}

The fatigue crack growth tests were performed in load control, at $550{ }^{\circ} \mathrm{C}$ on bars of Inconel 718, heat treated according to AMS 5663 standard, and with an approximate grain size of $10 \mu \mathrm{m}$, using Kb-type (surface crack) specimens with rectangular cross sections of $4.3 \times 10.2 \mathrm{~mm}$, see Fig. 1 . The initial notch was created by electro discharge machining $(\mathrm{EDM})$, and the subsequent pre-cracking was done at room temperature using a $10 \mathrm{~Hz}$ sine wave with a max load of $650 \mathrm{MPa}$ and $R_{\sigma}=0.05$. Crack growth was monitored by direct current potential drop (DCPD), while the subsequent stress intensity factor (SIF) evaluation [24] was done by assuming a semi-circular crack front. In all tests the point of consideration was the deepest point of the crack geometry, for which the cracked area was evaluated through a pre-defined calibration curve. The latter was based on temperature induced beach marks for cyclic load of $0.5 \mathrm{~Hz}$, for 
which measurements of crack length and PD values were correlated with each other. For additional description about the experiments see also [3, 25].

A list of the tests simulated here is found in Table 1, where the initial and final equivalent crack lengths are shown with the loading condition for each test. The three different test types consist of cyclic load (no environmentally associated damage), sustained load (cracking by environmentally-based damage), and finally a mix of both. The mixed test contains a block of cyclic load (to a crack length of $0.55 \mathrm{~mm}$ ) followed by a sustained load block (to a crack length of $1.8 \mathrm{~mm}$ ), and is finally ended by another cyclic block. Note that the PD method has an accuracy of only $0.01 \mathrm{~mm}$, which has been considered when evaluating the different tests, and that all load reversals were performed using a sine wave of $0.5 \mathrm{~Hz}$ and a max load of $650 \mathrm{MPa}$. The pre-defined calibration curve is derived for a relatively fast load $(0.5 \mathrm{~Hz})$, which generates time-independent behaviour for which only transgranular cracking is found. It is important to note that the considered load-bearing zone of each test specimen is the area not indicated by the PD reading. Likewise, the crack length that is used to derive the SIF in all the analyses below is defined by the area interpreted by PD.

Table 1: Tests simulated, with crack lengths according to post-mortem measurements (and PD evaluation).

\begin{tabular}{lll}
\hline Test type & $a_{0}[\mathrm{~mm}]$ & $a_{\text {final }}[\mathrm{mm}]$ \\
\hline Cyclic test $R=0.05$ & $0.23(0.23)$ & $2.41(2.41)$ \\
Cyclic test $R=0.6$ & $0.51(0.50)$ & $2.41(2.43)$ \\
Sustained load test & $0.48(0.49)$ & $2.94(2.50)$ \\
Mixed test & $0.24(0.24)$ & $2.56(2.59)$ \\
\hline
\end{tabular}

\subsection{Experimental results}

The crack growth rate results of the cyclic tests are seen in Fig. 2, while the results of the sustained load test and mixed test are seen in Figs. 3 and 4.

The cyclic tests have, as expected, more crack closure for the $R=0.05$ test than for $R=0.6$. Please note that the $R=0.05$ test was initiated with a short initial crack length, 
which gives the distinct ramp to the linear Paris region.

For the sustained load test and the mixed test it is noted an initial transient of the time dependent crack growth rate $\mathrm{d} a / \mathrm{d} t$ in Fig. 3, which indicates the build up of the damaged zone length, i.e. the length of weakened and cracked grain boundaries. When the stabilised crack growth rates are reached, the damaged zone has reached its stabilised level, at which it will be kept by the triaxial stress state of the test specimen. Investigation of the length of the damaged zone, which will be used for the modelling part of the paper is shown in Fig. 4, where data from the second block of the mixed test is used. The damaged zone, consisting of weakened and cracked grain boundaries is broken through as the rapid cyclic loading is applied, thus increasing the crack growth rate significantly. More details about the damaged zone concept and experimental findings including fracture surface investigations regarding the same can be found in e.g. [25].

\section{The basic 1D model}

The history-dependent model utilised in this paper takes its form from the evolution of material damage in front of the crack tip, see e.g. [26, 27]. The model, based on the damaged zone concept, is presented in Table 2, cf. [3,23], where the parameter $D$ (damaged zone length) is used for representing the history dependence. By describing its slow build-up during sustained loads, and rapid consumption during load reversals, an effective method of handling the history dependence is achieved. This also gives the possibility to directly relate the crack length physically measured on the fracture surface, and the crack length interpreted by the PD signal. Finally, the indexes " $t$ " and " $c$ " in Table 2 refer to the type of damage and crack growth behaviour, i.e. time dependent or cyclic.

In Table 2 the parameter $D_{\max }$ represents the maximum size of the damaged zone under stationary conditions, while $C_{0 t}, n_{t}, B_{t}, C_{c}, n_{c}, A_{c}$ and $B_{c}$ are fitting parameters. A crack closure routine[15], for effective handling of the different $R$ values is also introduced, 
which includes the constraint parameter $\alpha$ and the load ratio of maximum to flow stress, $\sigma_{\max } / \sigma_{0}$.

\subsection{Calibration of the $1 D$ model}

In order to handle the model calibration, the software LS-OPT [28] was used to minimise a least square-based error function. More specifically, the mixed test (sustained load part) and the sustained load test (cf. Table 1) were used to find values for $C_{0 t}$ and $n_{t}$, see Fig. 3, as well as for finding the fitting parameter $B_{t}$ (by a combined optimisation by the two tests). The cyclic power law parameters $C_{c}$ and $n_{c}$ were gained through linear regression (with closure correction), using the two cyclic tests, while $\sigma_{\max } / \sigma_{0}$ were set to 0.3 in accordance with recommendations found in [29] and $\alpha$ to 2.5 which was considered suitable for the geometry under consideration. The remaining parameters, $A_{c}, B_{c}$ and $D_{\max }$ were taken from the second cyclic block of the mixed test, see Fig. 4. All the parameter values are presented in Table 3.

\section{3D Modelling}

To extend the basic 1D model in the previous section to three dimensional crack growth, for application on general structural components, some major development has to be carried out. As the model has to be efficient and allow for applications in a general context, we have chosen to take an industrially recognised constraint method, usually used for 1D, and applied it to 3D. More specifically, we have used the constraint parameter $\alpha$ from the model by Newman [15], which has been successfully used in many commercial crack propagation programs, e.g. [29], and which is used in the 1D model described above. By this, we get an efficient method which is not so heavily dependent on extremely dense FE meshes for dissolving the actual constraint, and by which reasonably accurate results for 3D crack growth can be obtained. Below a description of the features of the modelling approach can be found. 


\subsection{Cyclic closure model}

A local crack closure procedure was implemented in order to achieve an accurate front description. As described above, the procedure uses the geometrical $\alpha$ value available in the Newman crack closure law [15], which takes the value 1 for complete plane stress- and 3 for complete plane strain conditions. The value is used as a scale function over the complete front with the use of a bell curve with optimised parameters, see e.g. [30]. The proposed description of the constraint does not only supply a direct method of translating the $1 \mathrm{D} \alpha_{\text {global }}$ value into $3 \mathrm{D}$ but also provides for $C^{1}$ continuity.

For the current test specimens, $\alpha_{\text {global }}=2.5$ was chosen (see the calibration of the $1 \mathrm{D}$ model above), which seems to fit the 1D crack growth rates reasonably well. The bell curve fitting parameters were then optimised together in order to achieve the same average/global $\alpha$ value as was chosen for the test specimen, cf. Eq. (1), with the parameters and results obtained shown in Table 4 and Fig. 5, respectively. The local value, $\alpha_{\text {local }}$, thus controls the crack length extension on a local level, but is still governed by the $1 \mathrm{D}$ optimised plane stress/strain state value based on experimentally gained crack growth rates of the specific test specimen. Note that the parameter $\varphi$ represents each point along the crack front stretching from 0 to $180^{\circ}$.

$$
\begin{aligned}
& f\left(\varphi ; E_{1}, E_{2}, E_{3}, E_{4}\right)=\frac{E_{4}}{1+\left|\frac{\varphi / \pi-E_{3}}{E_{1}}\right|^{2 E_{2}}} \\
& \alpha_{\text {local }}\left(\varphi ; E_{1}, E_{2}, E_{3}, E_{4}, E_{5}\right)=f\left(\varphi ; E_{1}, E_{2}, E_{3}, E_{4}\right)+E_{5} \\
& \alpha_{\text {global }}=\int_{0}^{\pi} \frac{\alpha_{\text {local }}\left(\varphi ; E_{1}, E_{2}, E_{3}, E_{4}, E_{5}\right)}{\pi} \mathrm{d} \varphi=2.5
\end{aligned}
$$


Table 4: Bell curve parameters for Eq. (1).

\begin{tabular}{ll}
\hline Parameter & Value \\
\hline$E_{1}$ & 0.44 \\
$E_{2}$ & 9.14 \\
$E_{3}$ & 0.5 \\
$E_{4}$ & 1.7 \\
$E_{5}$ & 1 \\
\hline
\end{tabular}

\subsection{Embrittling element damage control}

The same procedure as above was also applied for the local value (along the crack front) of the damaged zone extension, as the supply of embrittling elements depends on the local constraint, cf. the tunnelling of the cracks reported in e.g. $[9,10,17]$. For this procedure, the same optimised parameters as for the cyclic case were used, with the exception of it being scaled with the global damaged zone for $1 \mathrm{D}$. The value $D_{\text {global }}^{\max }=0.5$ $\mathrm{mm}$ was gained from the geometrical conditions of the specific test specimen, see Fig. 4 and Table 3. In order to perform this scaling procedure, an equivalent parameter to $E_{5}$ (see Table 4) for the cyclic load case has to be derived, here the parameter $E_{7}$ was used. The complete process of the above procedure is seen in Eq. (2), Table 5 and Fig. 6. By applying this procedure it is assumed that the constraint controls the cyclic as well as the sustained load damage, which is also physically motivated as both load modes are subjected to the same geometry. The local value $D_{\text {local }}^{\max }$ thus controls the maximum extent of the damaged zone exactly as $\alpha_{\text {local }}$ does for the cyclic crack growth.

$$
\begin{aligned}
& f\left(\varphi ; E_{1}, E_{2}, E_{3}, E_{6}\right)=\frac{E_{6}}{1+\left|\frac{\varphi / \pi-E_{3}}{E_{1}}\right|^{2 E_{2}}} \\
& D_{\text {local }}^{\max }\left(\varphi ; E_{1}, E_{2}, E_{3}, E_{6}, E_{7}\right)=f\left(\varphi ; E_{1}, E_{2}, E_{3}, E_{6}\right)+E_{7} \\
& D_{\text {global }}^{\max }=\int_{0}^{\pi} \frac{D_{\text {local }}^{\max }\left(\varphi ; E_{1}, E_{2}, E_{3}, E_{6}, E_{7}\right)}{\pi} \mathrm{d} \varphi=0.5
\end{aligned}
$$


Table 5: Bell curve parameters for Eq. (2).

\begin{tabular}{ll}
\hline Parameter & Value \\
\hline$E_{6}$ & 0.31 \\
$E_{7}$ & 0.22 \\
\hline
\end{tabular}

\subsection{Implementation}

The 3D analysis may be handled in a number of ways, the one chosen in this paper is a process where each crack front step is generated by a pre-defined global growth step. This value is then locally scaled with the normalised local SIF to the median SIF, thus creating the necessary information for the subsequent re-meshing step. Linear interpolation of the local SIF values is performed based on 101 front directions from 0 to $180^{\circ}$ (50 on each $90^{\circ}$ sector and 1 in the middle for symmetry purposes). These paths, with the local SIF in each direction, will thereafter be used for predicting the subsequent crack growth rate and e.g. crack length vs. time for model prediction. Details regarding the relations used for this procedure follow below.

\subsubsection{Front shape evolution}

The procedure in this paper is to assume that, during the cyclic part, the only parameters which are required to control the front shape is the local and global $\alpha$ value in the closure equation, see Eq. (3). For the sustained load mode, on the other hand, the shape evolution is assumed to be dependent on the ratio between the value of $D_{\text {local }}^{\max }$ and $D_{\text {global }}^{\max }$, as derived in Section 4.2, see Eq. (4) and Fig. 6. These simplifications will still provide for describing the constraint effects and will at the same time give the advantage of not having to predict the value of $D$ along each point, at each time step, along the complete crack front. The idea that the ratio between $D_{\text {local }}^{\max }$ and $D_{\text {global }}^{\max }$ should be the controlling parameter can of course be discussed but was chosen as a simple and easily defined principle. In Eqs. (3) and (4) the parameter $\Delta a_{\text {pre def. is the global incremental growth }}$ step. Note that the parameters $n_{c}$ and $n_{t}$ are the same as in Table 3 . 


$$
\begin{aligned}
& \frac{\mathrm{d} a}{\mathrm{~d} N}=C_{c}\left[\frac{1-f(\alpha, \ldots)}{1-R} \Delta K\right]^{n_{c}} \\
& \Delta a_{i}=\Delta a_{\text {pre def. }}\left(\frac{\frac{\mathrm{d} a}{\mathrm{~d} N}{ }_{i}\left(\Delta K_{i}, \alpha_{\text {local }}, \ldots\right)}{\frac{\mathrm{d} a}{\mathrm{~d} N}\left(\Delta K_{\text {median }}, \alpha_{\text {global }}, \ldots\right)}\right) \\
& \Delta a_{i}=\Delta a_{\text {pre def. }}\left(\frac{K_{i} \times \frac{D_{\text {local }}^{\text {max }}}{D_{\text {global }}^{\text {max }}}}{K_{\text {median }}}\right)^{n_{t}}
\end{aligned}
$$

The implementation of this model, although simple in form, requires an large amount of coding for efficient handling. All the equations have to be combined with the crack front evolution and its later growth history, and to achieve this, combined scripting based on FORTRAN, Python and Linux Bash was performed. The equations derived above were then used to predict the growth rates and related data, e.g. crack length vs. time.

\subsection{FE model}

In this work, the commercially available 3D crack growth program FRANC3D [31] in combination with Abaqus [32] was used, where the model of the Kb specimen was simplified as a rectangular block of cross section of $4.3 \times 10.2 \mathrm{~mm}$, with a height of 100 $\mathrm{mm}$, see Fig. 1 for test specimen details. To save time when re-meshing the crack front, only a portion of the model was selected to undergo this procedure, from the selected model a $4.3 \times 10.2 \times 20 \mathrm{~mm}$ sub-model was extracted. The method of FRANC3D used in this paper (briefly explained) was to initiate a crack by specified front coordinates which was then propagated according to the scheme for each growth mode, as described above. For each growth step, the crack front was re-meshed by three rings of elements, where the inner one consists of 15 node wedge elements with nodes moved to L/4 for $1 / \sqrt{r}$ strain singularity and the outer ones of 20 node brick elements. The bulk mesh in the sub-model consists of 10 node tetrahedral elements. For solving the SIFs (built into FRANC3D) the interaction integral was used, see e.g. [33]. 
The choice of element size for the elements closest to the crack front was based on the current crack length, and set to about $10 \%$ of it according to a mean value of the current front positions. The SIFs were extracted and used together with the specified growth model as explained in the above section, and for this procedure the pre-defined crack

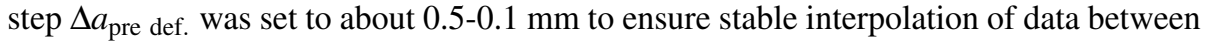
the different front positions. After the re-meshing of the crack front, the sub-model was put back into the global model (also consisting of 20 node brick elements) and solved in Abaqus. In Fig. 7 a typical mesh is seen which consists of about 67500 elements and 112000 nodes.

\subsubsection{Boundary conditions}

The model was restrained to let plane surfaces remain plane and not being able to tilt, which was achieved by multi-point constraint equations. A load of $650 \mathrm{MPa}$ was also applied, giving the ability to later on scale it to what was used in each specific test.

\subsection{Modified growth rate law}

The final step is to process the subsequent crack propagation rate, i.e. to incorporate the different growth steps into the growth law in Table 2. Every equation which in the 1D model contained $D_{\max }$ is modified to $D_{\text {local }}^{\max }$ instead, and when $D_{\text {local }}$ has reached $D_{\text {local }}^{\max }$ the maximum growth rate of the equations will be reached. The consequence will be that each front position will aim at exactly the same $D_{\text {local }}^{\max }$ value as the bell curve has specified. A complete model of the 3D stage to control the growth rate is seen in Table 6.

\subsection{Implementation summary}

A flow chart of the whole procedure is provided in Fig. 8. Initially an FE model of the structure is inserted with an initial flaw, which is meshed in FRANC3D and run in Abaqus. Subsequent SIFs are then calculated based on the results from the simulation. 
From the results one can, if the crack length has reached the final value, calculate crack growth rates by the model of Table 6 . One has to first decide whether cyclic or dwell time is active; for cyclic loads the front points $\Delta a_{i}$ are moved according to Eq. (3) and for dwell times according to Eq. (4). Each local front point is then translated the computed distance, a new crack front is defined, and the process starts over once again.

\section{Results and discussion}

It is to be noted that the results and discussion found below focus on the comparison between the simulated and experimentally found crack front evolution, as more global results regarding hold time effects, such as $\mathrm{d} a / \mathrm{d} N$ and $\mathrm{d} a / \mathrm{d} t$ diagrams, have been reported elsewhere, see e.g. [3].

The cyclic tests are shown in Fig. 9 for $R_{\sigma}=0.05$ and in Fig. 10 for $R_{\sigma}=0.6$, where the different final crack lengths can be seen to be captured realistically. More closure of the test for $R_{\sigma}=0.05$ can also be noted by slightly more tunnelling behaviour at the surface, but is seen to give some discrepancy from the actual test. On the other hand for the $R_{\sigma}=0.6$ test the crack front is captured accurately and almost no test to model difference can be seen.

For the front evolution of the sustained load test, the model of the damaged zone growth controls the embrittlement element supply. The ratio of constraint from the cyclic model which was transferred to the sustained load model, captures the tunnelling effect with reasonable accuracy and gives a correct front evolution, see Fig. 11. The end marking of the final damaged zone length, from Fig. 6, is also shown (extrapolated from the final front value) and clearly shows how much the sustained load influences tunnelling on the fracture surface.

The final test with the mixed load mode, see Fig. 12, shows the interaction properties of dwell time and cyclic load. First with the cyclic block there is almost no tunnelling (in accordance with Fig. 9). Then, the sustained load block with the embrittling element damage control law is applied (increased tunnelling effect), followed by the last block 
of cyclic load where the crack front evolution yet again takes the form of a semi-circular shape. This demonstrates how the history effects of the different load modes benefit from the model, and how it can accurately track the front evolution.

Simulation results for crack length vs. time of the implemented model, compared to the test results are summarised in Table 7. Note that discrepancy originates from some different reasons, the first is that the tests are evaluated based on 1D, i.e. a semi-circular crack front, another reason is that the elementary case used for the evaluation of the mechanical experiments is not as in the machine, see [24]. In the elementary case, there is no bending restriction, i.e. long cracks might be subjected to some bending from the tensile stress. Other reasons can be originating from the FE boundary conditions in this paper, which were made to assure that only tensile stresses were given. For relatively short cracks (as in the case here) this should not present any major problem. Finally, it should be noted that the SIF values related to the evaluation paths closest to the test specimen border have been disregarded due to bad quality in interpolating them.

Table 7: Time to final crack length, test and simulation results in hours.

\begin{tabular}{llll}
\hline Test type & Test & Developed model & $t_{\text {exp. }} / t_{\text {sim. }}$ \\
\hline Cyclic $R=0.05$ & 8.8 & 5.5 & 1.59 \\
Cyclic $R=0.6$ & 17.1 & 13.1 & 1.32 \\
Sustained load & 97.6 & 87.2 & 1.12 \\
Mixed & 81.2 & 73.3 & 1.10 \\
\hline
\end{tabular}

\section{Summary and conclusions}

The history dependent crack growth model in $[3,23]$ for $1 \mathrm{D}$ has been extended to a fully 3D context. The model is developed to provide for efficient analysis of 3D crack growth without having to depend on describing constraint effects by high density FE simulations. Simulations of surface cracks have been carried out for a number of different load modes for the Ni-based superalloy Inconel 718 at $550{ }^{\circ} \mathrm{C}$. The modelling concept is focused on the constraint differences along the crack front, for which a local closure description for 
the cyclic load part and an embrittling element damage control for the sustained load part are introduced. The results show that this concept of constraint description appears to be suitable for further applications, e.g. in component design.

\subsection{Suggestions for further improvement}

Regarding the front evolution, some simplifications were used. It was assumed that the bell curves in Figs. 5 and 6 are only based on the maximum values of the damaged zone. In reality the front description is affected in both cyclic and dwell time growth by the current length of the zone, which can be seen in Figs. 3 and 4. Creating such a front update scheme would improve the accuracy with respect to both the front shape and the subsequent crack growth rate, but can in its implementation require substantial recourses in order to function properly.

\section{Acknowledgements}

The authors would like to thank Mr. Patrik Härnman and Mr. Bo Skoog, Linköping University, for the laboratory work and the project teams at Linköping University, Siemens Industrial Turbomachinery AB and GKN Aerospace Engine Systems for valuable discussions. This research has been funded by the Swedish Energy Agency, Siemens Industrial Turbomachinery AB, GKN Aerospace Engine Systems, and the Royal Institute of Technology through the Swedish research programme TURBO POWER, the support of which is gratefully acknowledged.

\section{References}

[1] T. Nicholas, T. Weerasooriya, Hold-time effects in elevated temperature fatigue crack propagation, Fracture Mechanics: Seventeenth Volume ASTM STP 905 (1986) 155-168. 
[2] J. Gayda, T.P. Gabb, R.V. Miner, Fatigue crack propagation of nickel-base superalloys at $650^{\circ} \mathrm{C}$, Low Cycle fatigue ASTM STP 942 (1988) 293-309.

[3] E. Lundström, K. Simonsson, D. Gustafsson, T. Månsson, A load history dependent model for fatigue crack propagation in Inconel 718 under hold time conditions, Engineering Fracture Mechanics 118 (2014) 17-30.

[4] J.P. Pédron, A. Pineau, The effect of microstructure and environment on the crack growth behaviour of Inconel 718 alloy at $650^{\circ} \mathrm{C}$ under fatigue, creep and combined loading, Material Science and Engineering 56(2) (1982) 143-56.

[5] D. Bika, J.A. Pfaendtner, M. Menyhard, C.J. McMahon Jr., Sulfur-induced dynamic embrittlement in a low-alloy steel, Acta Metallurgica et Materialia 43(5) (1995) 1895-908.

[6] J.A. Pfaendtner, C.J. McMahon Jr., Oxygen-induced intergranular cracking of a Ni-base alloy at elevated temperatures - an example of dynamic embrittlement, Acta Materialia 49(16) (2001) 3369-3377.

[7] D.A. Woodford, Gas phase embrittlement and time dependent cracking of nickel based superalloys, Energy Materials: Materials Science and Engineering for Energy Systems 1(1) (2006) 59-79.

[8] U. Krupp, Dynamic embrittlement - time-dependent quasi-brittle intergranular fracture at high temperatures, International Materials Reviews 50(2) (2005) 83-97.

[9] F. V. Antunes, J. M. Ferreira, C. M. Branco, J. Byrne, Influence of stress state on high temperature fatigue crack growth in Inconel 718, Fatigue \& Fracture of Engineering Materials \& Structures (2001) 24(2) 127-135.

[10] P. Heuler, E. Affeldt, R.J.H. Wanhill, Effects of loading waveform and stress field on high temperature fatigue crack growth of alloy 718, Materialwissenschaft und Werkstofftechnik 34(9) (2003) 790-796.

[11] J.C. Newman Jr., C.A. Bigelow, K.N. Shivakumar, Three-dimensional elasticplastic finite element analyses of constraint variations in cracked bodies, Engineering Fracture Mechanics 46 (1993) 1-13. 
[12] M.L. Zhu, F.Z. Xuan, S.T. Tu. Observation and modeling of physically short fatigue crack closure in terms of in-situ SEM fatigue test. Materials Science and Engineering: A, 618 (2014) 86-95.

[13] J.P. Tan, S.T. Tu, G.Z. Wang, F.Z. Xuan. Characterization and correlation of 3-D creep constraint between axially cracked pipelines and test specimens. Engineering Fracture Mechanics, 1362015 96-114.

[14] M.L Zhu, F.Z Xuan, S.T. Tu. Effect of load ratio on fatigue crack growth in the near-threshold regime: A literature review, and a combined crack closure and driving force approach. Engineering Fracture Mechanics, 141 (2015) 57-77.

[15] J.C. Newman Jr., A crack opening stress equation for fatigue crack growth, International Journal of Fracture 24 (1984) R131-R135.

[16] M.A. James, J.C. Newman Jr., The effect of crack tunneling on crack growth: Experiments and CTOA analyses, Engineering Fracture Mechanics 70(3-4) (2003) 457-468.

[17] J. Tong, T-stress and its implications for crack growth, Engineering Fracture Mechanics 69 (2002) 1325-1337.

[18] J.C. Newman Jr., I.S. Raju, Prediction of fatigue crack-growth patterns and lives in three-dimensional cracked bodies, Proceedings of the 6th International Conference on Fracture (ICF6) (1984) 1597-1608.

[19] W.U. Shang-Xian, Shape evolution of surface crack during fatigue growth, Engineering Fracture Mechanics 22 (1985) 897-913.

[20] F. Nilsson, A few parameter crack growth description procedure, International Journal of Fracture 54 (1992) 35-44.

[21] R. Branco, F.V. Antunes, L.C.H. Ricardo, J.D Costa, Extent of surface regions near corner points of notched cracked bodies subjected to mode-I loading, Finite Elements in Analysis and Design 50 (2012) 147-160. 
[22] P.F.P. de Matos, D. Nowell, Numerical simulation of plasticity-induced fatigue crack closure with emphasis on the crack growth scheme: 2D and 3D analyses, Engineering Fracture Mechanics 75 (2008) 2087-2114.

[23] E. Lundström, K. Simonsson, T. Månsson, D. Gustafsson, Modelling of fatigue crack growth in Inconel 718 under hold time conditions - application to a flight spectrum,Advanced Materials Research 891-892 (2014) 759-764.

[24] J.C. Newman Jr., I.S. Raju, Stress-intensity factor equations for cracks in threedimensional finite bodies subjected to tension and bending loads, NASA Technical Memorandum 85793 (1984).

[25] E. Storgärds, K. Simonsson, Crack length evaluation for cyclic and sustained loading at high temperature using potential drop, Experimental Mechanics 55(3) (2015) 559-568.

[26] K.-M. Chang, M.F. Henry, and M.G. Benz, Metallurgical control of fatigue crack propagation in superalloys, Journal of Metals 42(12) (1990) 29-35.

[27] S.Y. Yu, H.Y. Li, M.C. Hardy, S.A. McDonald, P. Bowen, Mechanisms of dwell fatigue crack growth in an advanced nickel disc alloy RR1000, 2nd European Symposium on Superalloys and Their Applications, EUROSUPERALLOYS 2014 14 (2014).

[28] N. Stander, W. Roux, T. Goel, T. Eggleston, K. Craig, LS-OPT User's manual, Version 4.2, Livermore Software Technology Corporation, Livermore, CA (2011).

[29] NASGRO manual 7.0, SwRI.

[30] MATLAB, version 8.2.0.701 (R2013b), The MathWorks Inc., Natick, Massachusetts 2013 .

[31] FRANC3D manual 6.0, Fracture Analysis Consultants, Inc.

[32] Abaqus theory manual 6.12, Dassault Systémes Simulia Corp., Providence 2012.

[33] M. Kuna, Finite Elements in Fracture Mechanics Theory-Numerics-Applications, Springer 2013. 
Time dep. crack growth rate:

Time dep. scale function:

$$
\begin{aligned}
& \dot{a}_{t}=\frac{D}{D_{\max }} \cdot C_{t} K_{\max }^{n_{t}} \\
& C_{t}=C_{0 t}\left(1-\exp \left(\frac{-B_{t}}{1-\frac{D}{D_{\max }}}\right)\right) \quad B_{t} \geq 0
\end{aligned}
$$

Time dep. damaged zone growth rate:

$$
\dot{D}_{t}=\dot{m}-\dot{a}_{t}
$$

Damage mechanism based growth rate: $\quad \dot{m}=C_{t} K_{\max }^{n_{t}}$

Cyclic crack growth:

$$
\begin{aligned}
& \Delta a_{c}=\int_{t_{1}}^{t_{2}} \dot{a}_{c} \mathrm{~d} t=\int_{0}^{1} \frac{\mathrm{d} a_{c}}{\mathrm{~d} N} \mathrm{~d} N \\
& \frac{\mathrm{d} a_{c}}{\mathrm{~d} N}=S_{c} \cdot C_{c}\left[\frac{1-f}{1-R} \Delta K\right]^{n_{c}} \\
& f=\frac{K_{o p}}{K_{\max }}= \begin{cases}\max \left(R, A_{0}+A_{1} R+A_{2} R^{2}+A_{3} R^{3}\right) & -2 \leq R<0 \\
A_{0}+A_{1} R & \end{cases} \\
& A_{0}=\left(0.825-0.34 \alpha+0.05 \alpha^{2}\right)\left[\cos \left(\pi \sigma_{\max } / 2 \sigma_{0}\right)\right]^{1 / \alpha} \\
& A_{1}=(0.415-0.071 \alpha) \sigma_{\max } / \sigma_{0} \\
& A_{2}=1-A_{0}-A_{1}-A_{3} \\
& A_{3}=2 A_{0}+A_{1}-1
\end{aligned}
$$

Cyclic damaged zone evolution:

$$
\begin{aligned}
\Delta D_{c} & =\int_{t_{1}}^{t_{2}} \dot{D}_{c} \mathrm{~d} t=\int_{0}^{1} \frac{\mathrm{d} D_{c}}{\mathrm{~d} N} \mathrm{~d} N \\
\frac{\mathrm{d} D_{c}}{\mathrm{~d} N} & =-\frac{\mathrm{d} a_{c}}{\mathrm{~d} N}
\end{aligned}
$$

Cyclic scale function:

Table 3: Model parameters, units in $\mathrm{MPa}, \mathrm{MPa} \sqrt{\mathrm{m}}, \mathrm{m} / \mathrm{s}$ and $\mathrm{m} /$ cycle; $D_{\max }$ in $\mathrm{mm}$.

\begin{tabular}{llllllllll}
\hline$C_{0 t}$ & $n_{t}$ & $C_{c}$ & $n_{c}$ & $B_{t}$ & $A_{c}$ & $B_{c}$ & $D_{\max }$ & $\alpha$ & $\sigma_{\max } / \sigma_{0}$ \\
\hline $2.6 \times 10^{-13}$ & 3.3 & $3.3 \times 10^{-10}$ & 2.3 & 1.45 & 331 & 2.7 & 0.5 & 2.5 & 0.3 \\
\hline
\end{tabular}


Time dep. effective $K$ :

Time dep. crack growth rate:

Time dep. scale function:

Time dep. damaged zone growth rate:

Damage mechanism based growth rate:

Cyclic effective $K$ :

Cyclic crack growth:

Cyclic scale function:

Cyclic damaged zone evolution:

$$
\begin{aligned}
& K_{\text {max eff. }}=K_{\text {max }} \times D_{\text {local }}^{\max } / D_{\text {global }}^{\max } \\
& \dot{a}_{t}=\frac{D_{\text {local }}}{D_{\text {local }}^{\max }} \cdot C_{t} K_{\text {max eff. }}^{n_{t}} \\
& C_{t}=C_{0 t}\left(1-\exp \left(\frac{-B_{t}}{1-\frac{D_{\text {local }}}{D_{\text {local }}^{\max }}}\right)\right) \quad B_{t} \geq 0
\end{aligned}
$$

$\dot{D}_{\text {local }_{t}}=\dot{m}-\dot{a}_{t}$

$\dot{m}=C_{t} K_{\max }^{n_{t}}$ eff.

$$
\Delta K_{\text {eff. }}=\left(1-f\left(\alpha_{\text {local }}, \ldots\right) /(1-R)\right) \Delta K
$$$$
\Delta a_{c}=\int_{t_{1}}^{t_{2}} \dot{a}_{c} \mathrm{~d} t=\int_{0}^{1} \frac{\mathrm{d} a_{c}}{\mathrm{~d} N} \mathrm{~d} N
$$$$
\frac{\mathrm{d} a_{c}}{\mathrm{~d} N}=S_{c} \cdot C_{c}\left(\Delta K_{\text {eff. }}\right)^{n_{c}}
$$

$$
\begin{aligned}
& \Delta D_{\text {local }_{c}}=\int_{t_{1}}^{t_{2}} \dot{D}_{\text {local }_{c}} \mathrm{~d} t=\int_{0}^{1} \frac{\mathrm{d} D_{\text {local }_{c}}}{\mathrm{~d} N} \mathrm{~d} N \\
& \frac{\mathrm{d} D_{\text {local }_{c}}}{\mathrm{~d} N}=-\frac{\mathrm{d} a_{c}}{\mathrm{~d} N} \\
& S_{c}=1+A_{c}\left(\frac{D_{\text {local }}}{D_{\text {local }}^{\text {max }}}\right)^{B_{c}} \quad A_{c}, B_{c} \geq 0
\end{aligned}
$$



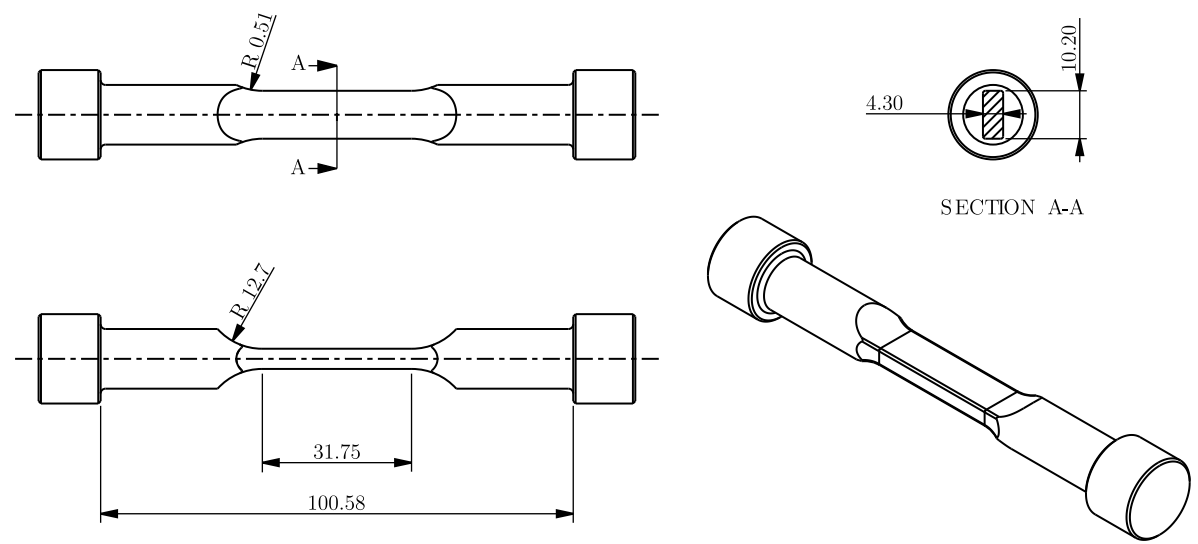

Figure 1: Drawing of $\mathrm{Kb}$-specimen with the rectangular cross section, dimensions in $\mathrm{mm}$. 


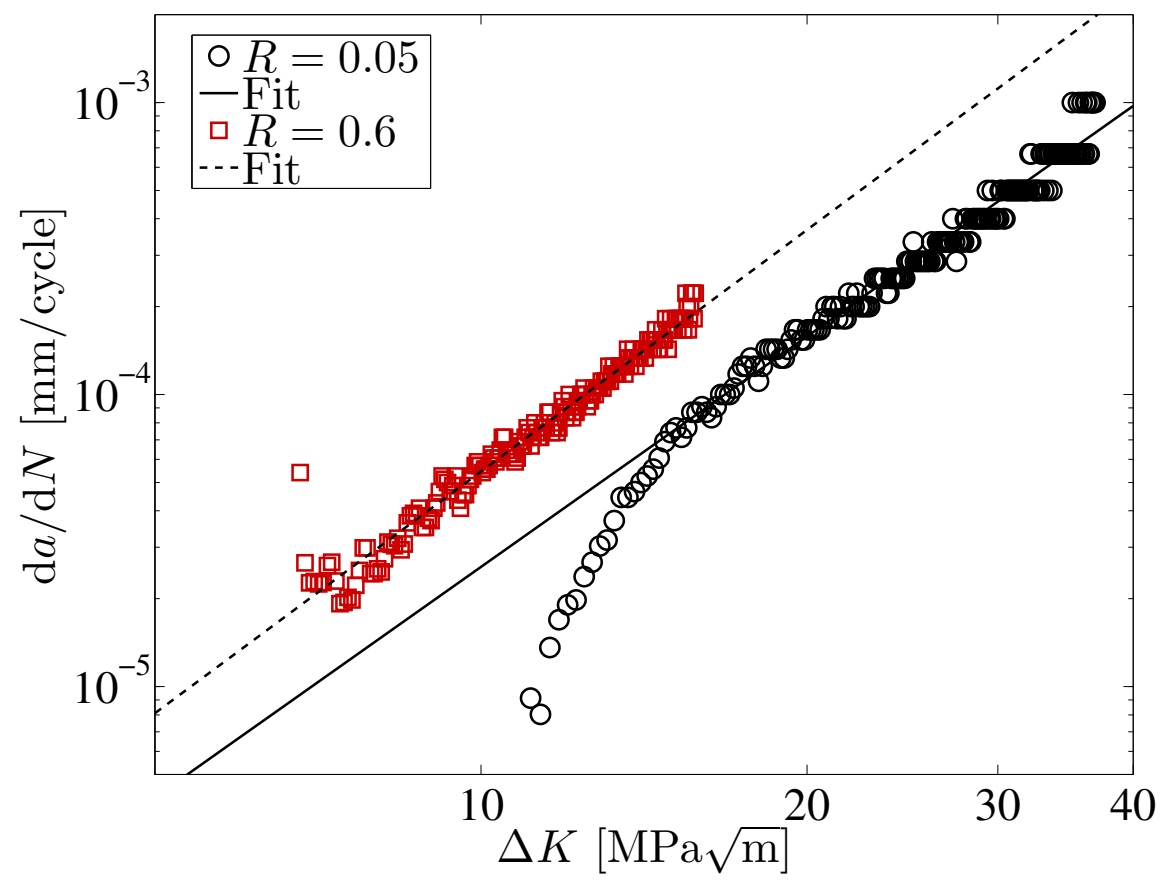

Figure 2: Crack growth rate for the cyclic tests. 


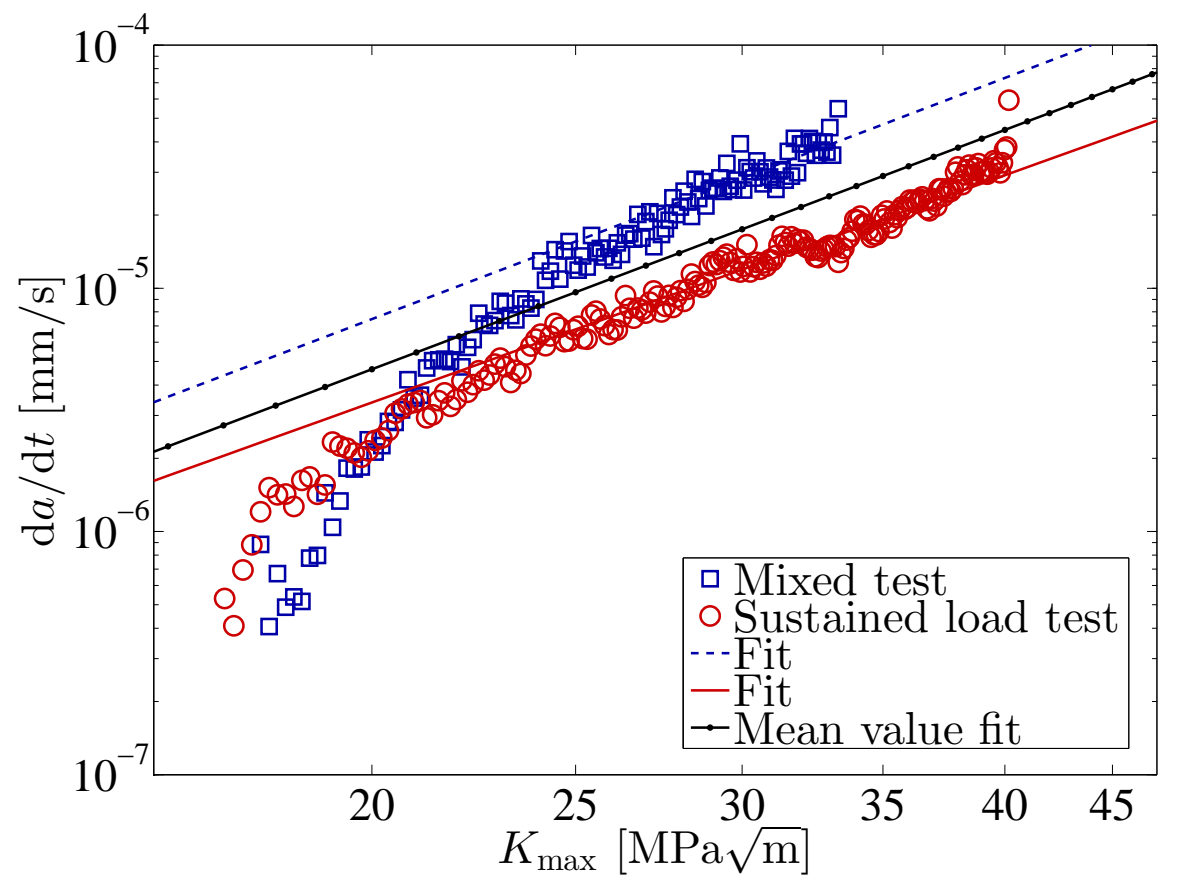

Figure 3: Time dependent crack growth rate for the sustained load parts of the dwell time test and the mixed test, also for calibration of $C_{0 t}, n_{t}$ and $B_{t}$. 


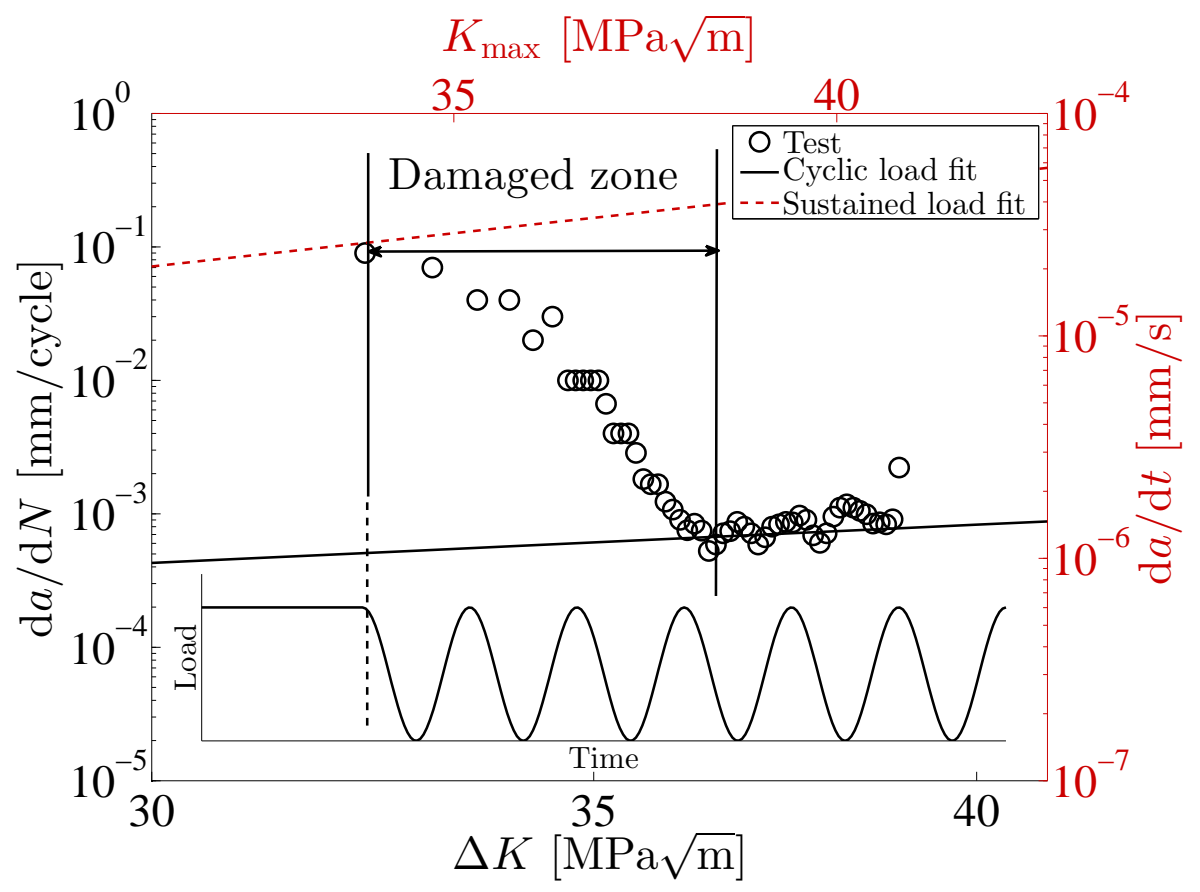

Figure 4: Second cyclic block of the mixed test at which the crack growth rate increases, the parameters $A_{c}$ and $B_{c}$ found by optimisation, while $D_{\max }$ is measured. 


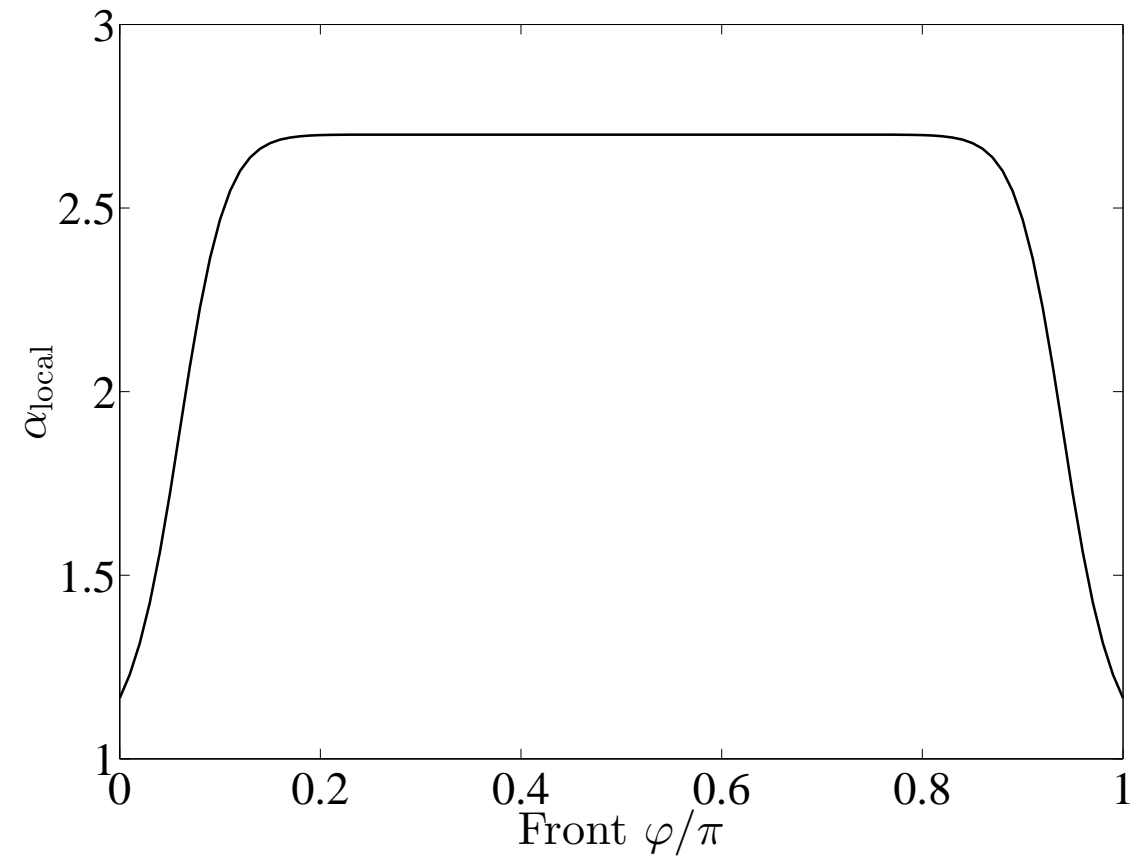

Figure 5: Bell curve for the cyclic part, local $\alpha$ value over the front to control the constraint relation. 


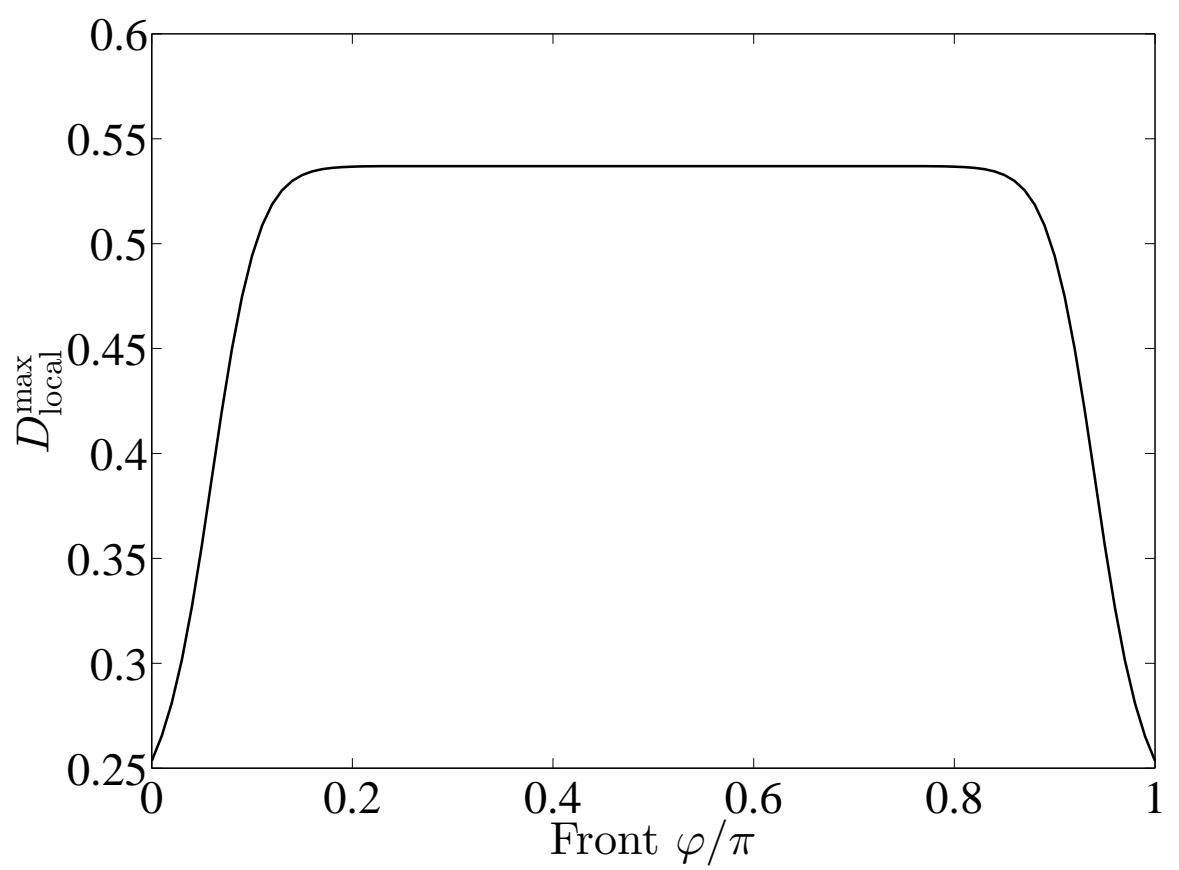

Figure 6: Bell curve for the sustained load part, local $D$ max values, $D_{\text {local }}^{\max }$, over the front to control the embrittling element supply. 


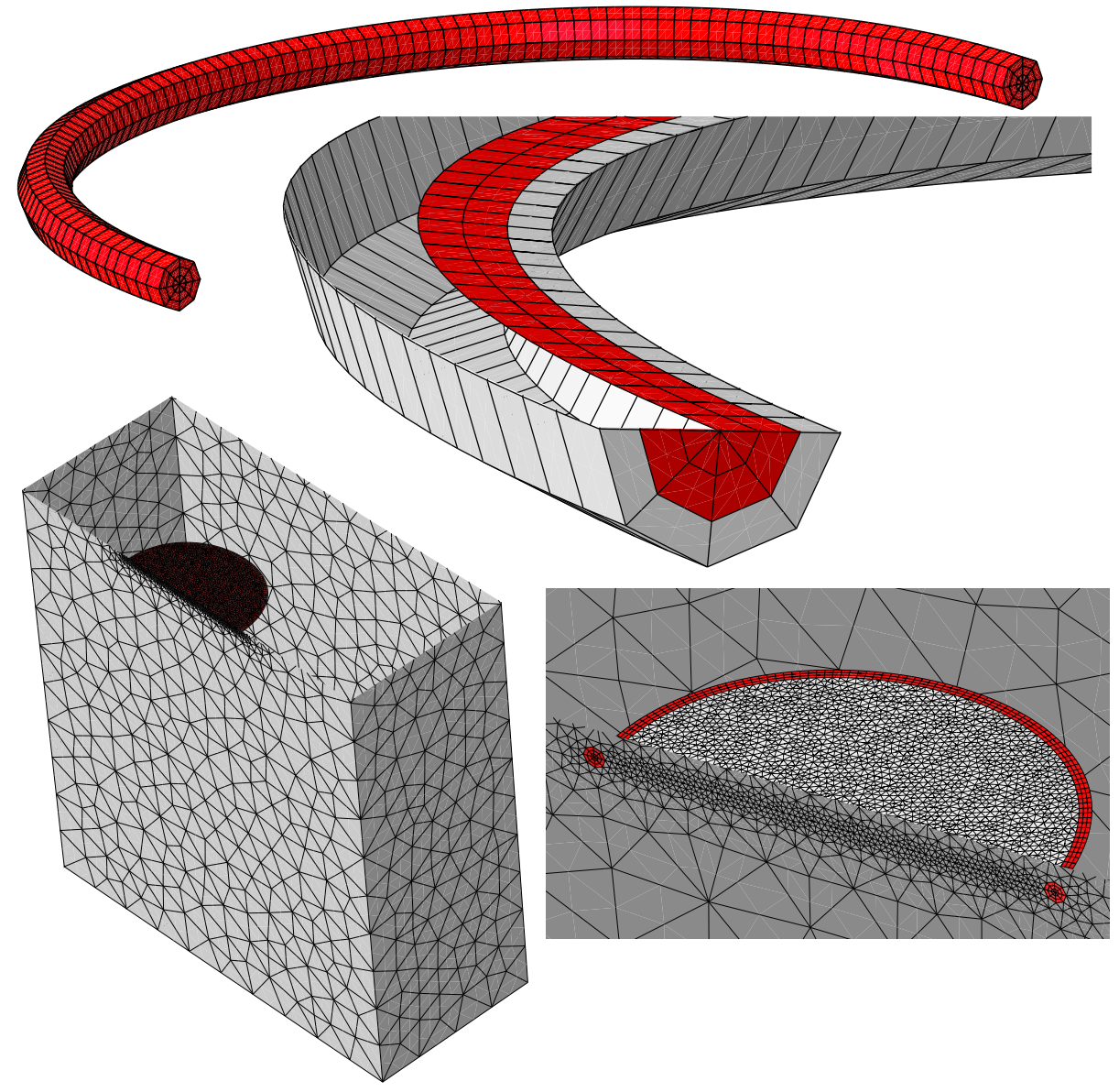

Figure 7: Mesh with the sub-model, extracted sub-model, initiated crack, template around the crack front and marked rings of elements for calculation of SIFs. 


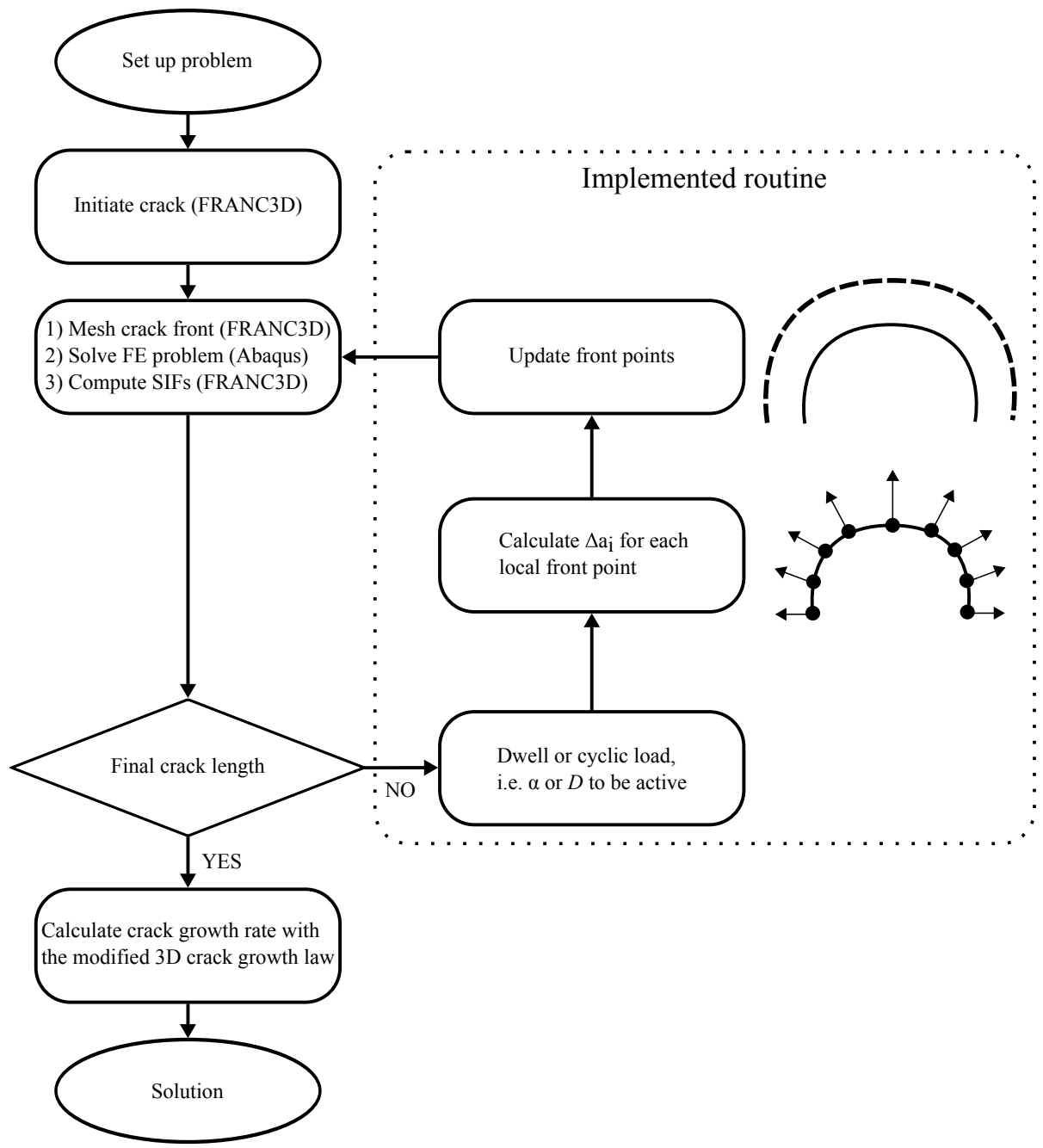

Figure 8: Flow chart of the complete procedure; an initial FE model is inserted with a crack which propagates according to the developed model, when the final crack length is reached the crack growth rate properties are calculated according to Table 6 . 


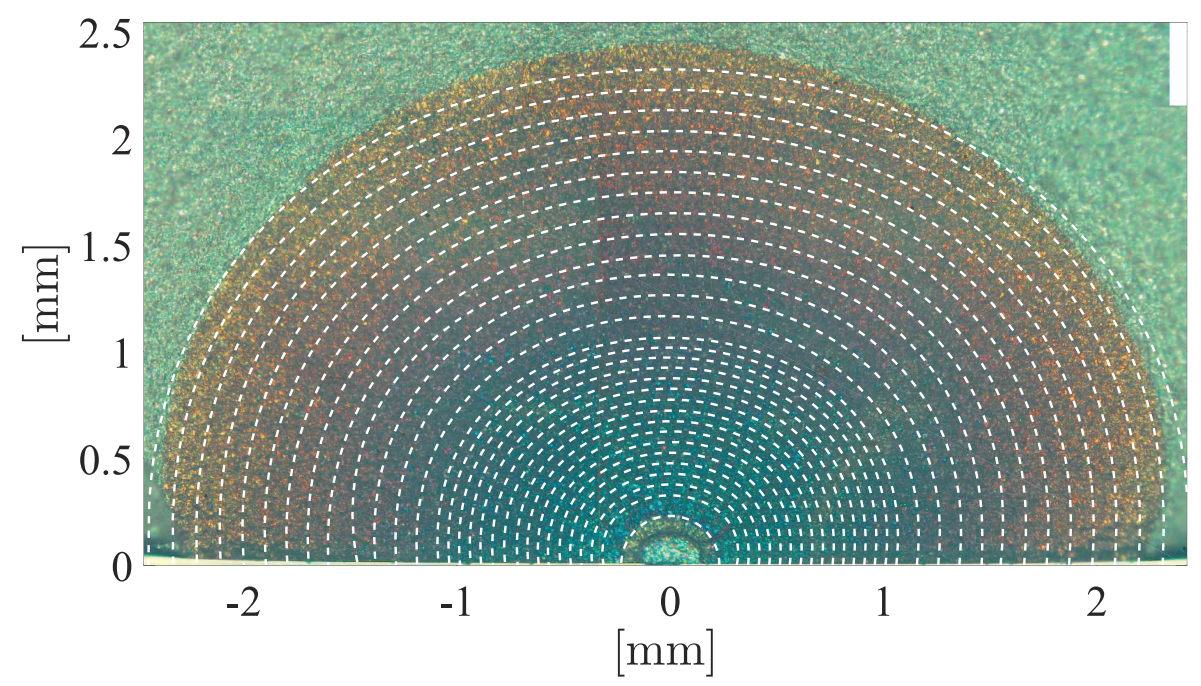

Figure 9: Cyclic test results for $R_{\sigma}=0.05$. Crack front shapes in white from the numerical simulations on fracture surface from the mechanical tests. 


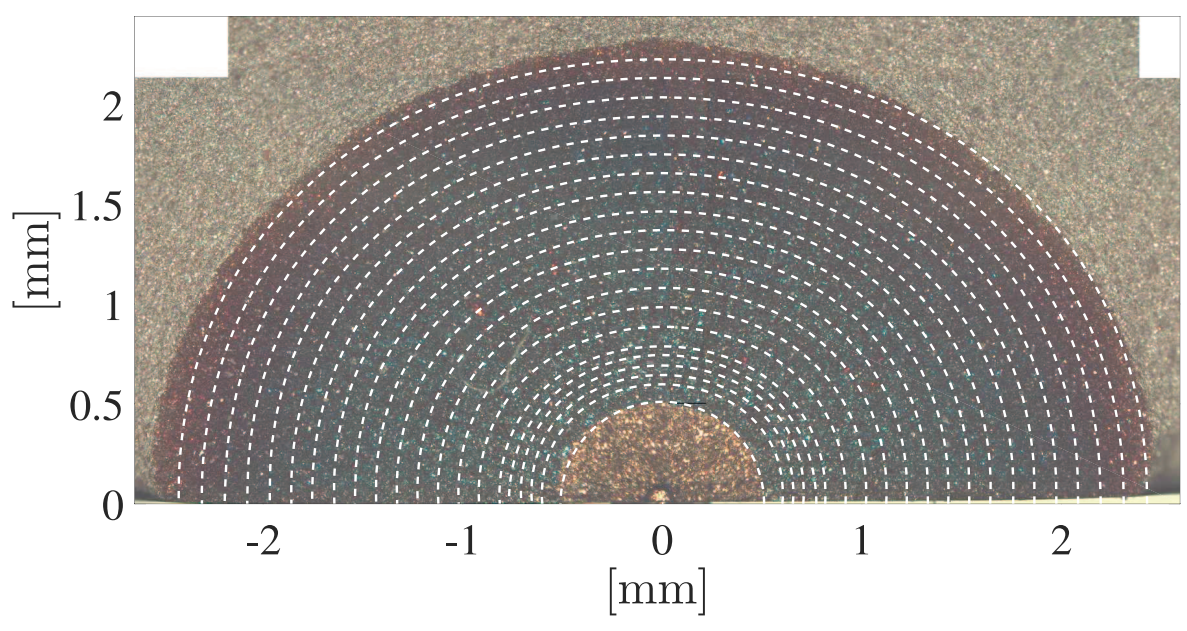

Figure 10: Cyclic test results for $R_{\sigma}=0.6$. Crack front shapes in white from the numerical simulations on fracture surface from the mechanical tests. 


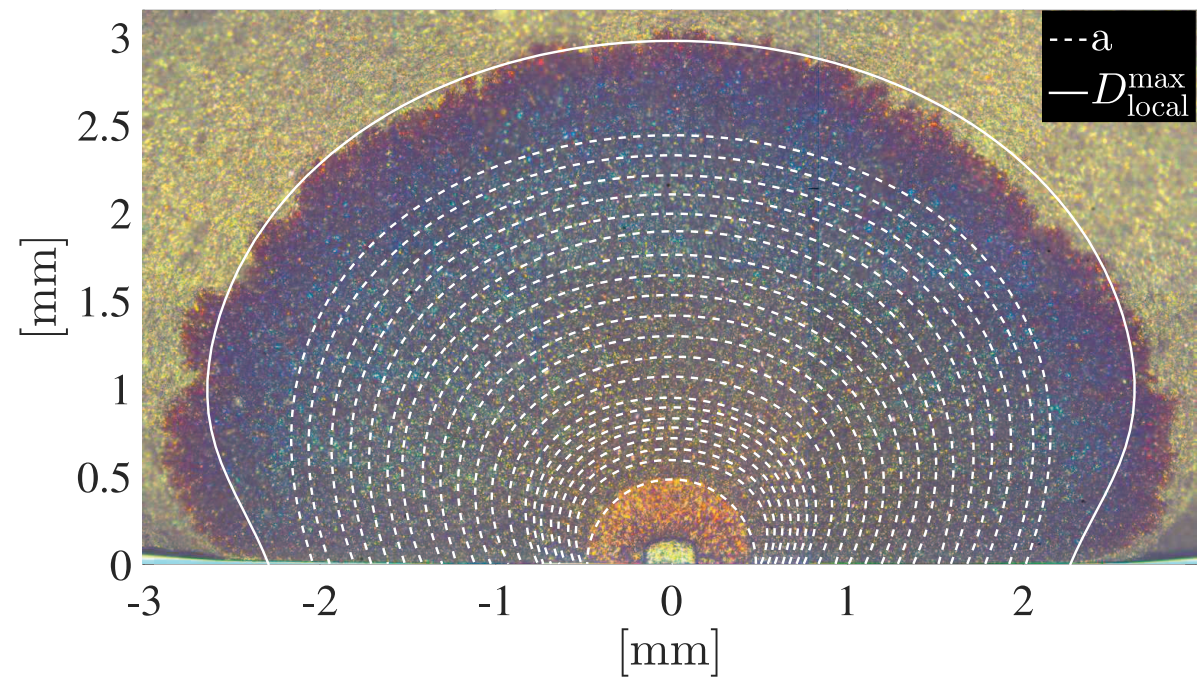

Figure 11: The sustained load test, numerical results in white with the mechanical test's fracture surface. The markings also include the final damaged zone marking, $D_{\text {local }}^{\max }$, extrapolated from the final crack length. 


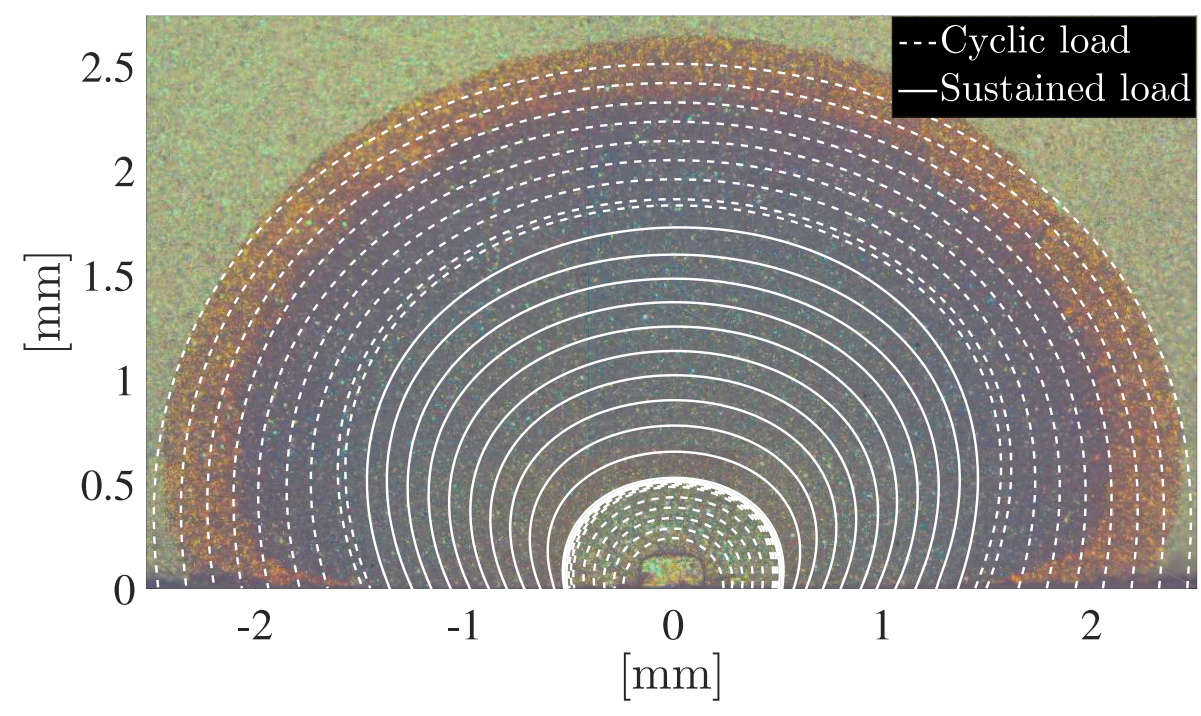

Figure 12: The mixed test result, numerical simulations on white with respective fracture surface in the background. Tunnelling interaction between the sustained and cyclic load is seen to vary depending on applied load block. 